\title{
Scanning electron microscopic studies of Beauveria bassiana against Lipaphis erysimi Kalt
}

\author{
Tanvi Sharma, Neelam Joshi* and Anu Kalia \\ Department of Microbiology, Punjab Agricultural University, Ludhiana-141004 (Punjab), INDIA \\ Department of Entomology, Punjab Agricultural University, Ludhiana-141004 (Punjab), INDIA \\ Department of Soil Science, Punjab Agricultural University, Ludhiana-141004 (Punjab), INDIA \\ *Corresponding author. E- mail: neelamjoshi_01@pau.edu
}

Received: July 4, 2016; Revised received: December 15, 2016; Accepted: February 14, 2017

\begin{abstract}
This work was aimed to identify the $\mathrm{LC}_{50}$ of the indigenous fungal isolates for controlling $L$. erysimi infestation in mustard aphid besides to probe the mechanism of action of the local isolates and comparison of the efficacy with the reference culture and commercial formulation 'Mycojaal'. Three isolates of entomopathogenic fungi Beauveria bassiana were tested for infection on nymph of Lipaphis erysimi Kalt. using scanning electron microscopy (SEM) to record any variation. The SEM revealed adhesion of spores of B.bassiana followed by penetration of L.erysimi nymph surface. It was observed that all Beauveria isolates showed little variation with respect to penetration and adhesion at different time intervals. Further, lethal concentration $\left(L_{50}\right)$ values of $B$.bassiana isolates against $L$. erysimi was recorded and was lowest $\left(0.05 \times 10^{7}\right.$ spores $\left./ \mathrm{ml}\right)$ in $B$. bassiana MTCC 4495 and highest $\left(0.11 . \times 10^{7}\right.$ spores $\left./ \mathrm{ml}\right)$ was recorded in native isolate $\mathrm{F} 10$ after 120 hours of treatment. The study has established the need for the isolation and evaluation of the indigenous Beauveria isolate. Moreover, it also exhibited the efficacy of the reference and commercially available biocontrol agents.
\end{abstract}

Keywords: Beauveria bassiana, Lipaphis erysimi, Lethal concentration, Scanning electron microscopy

\section{INTRODUCTION}

Mustard aphid (Lipaphis erysimi Kalt.) (Homoptera, Aphididae) is a major pest of brassica crops in subtropics and tropical regions. It feeds by sucking the sap of their host-plants and leads to leaf curling, shrivelling and yellowing, which affects the leaf size and yield. Mustard aphid may damage the crop from seedling stage to maturity, with highest population occurring during the flowering and podding stages. It is responsible for causing the yield losses ranging from 35.4 to 96 per cent (Sahoo, 2012). The chemical insecticides used to manage this pest cause environmental and health problems apart resulting in development of resistant. Indiscriminate use of synthetic chemical pesticides in crop protection causes several socioeconomic problems throughout the world. Harrington and Emden (2007) have shown development of resistance in mustard aphid to a number of carbamate, pyrethroid and organophosphate based insecticides. Aphid resistance to common insecticides has stimulated interest in developing alternative methods for its control for sustainable crop practices (Gurret al., 2004).

Entomopathogenic fungi are important biocontrol agents which play a vital role in pest management (Cooke, 1977). Various entomopathogenic fungi such as Lecanicillium sp. (Jung et al., 2006), B. bassiana (Quesada-Moraga et al., 2006), M. anisopliae, Isaria sp. and Nomuraea sp. (Devi et al., 2003) have been used for the management of insect pests. Among these entomopathogenic fungi, Beauveria species are the most promising biocontrol agents and are known to attack a wide range of insect pests. Worldwide, B.bassiana parasitizes arthropod species, causing white muscardine disease. This fungus rarely infects humans or other animals, which makes it a safer biocontrol agent for management of crop insects (Ormond et al., 2010). Keeping this in mind, the present study was conducted to compare pathogencity of three Beauveria isolates against nymph of $L$. erysimi and the SEM studies were performed to probe the mechanism of action of the local isolates as compared to commercial and reference strains.

\section{MATERIALS AND METHODS}

Source of Beauveria bassiana: Three Beauveria bassiana isolates, viz., B.bassiana MTCC 4495 (procured from IMTECH, Chandigarh), one native B.bassiana isolate F10 (isolated from soil sample by Galleria bait method) and one isolated from commercial formulation (Mycojaal, Pest control India, Bangalore) were evaluated in the present study. These isolates were maintained on Sabouraud Dextrose Yeast Extract Agar (SDYA) medium at refrigeration temperature.

Preparation of culture suspension: The SDYA medium was inoculated with the B.bassiana isolates and 
incubated at $25 \pm 2{ }^{\circ} \mathrm{C}$ in Erlenmeyer flasks under static conditions. After ten days of incubation the aqueous spore suspensions were prepared. The conidia were harvested by scrapping and suspended in sterilized 0.1 $\%$ Tween 80 solution (Luz et al.,1998)

Collection of $\boldsymbol{L}$. erysimi and bioassay: Adults and nymphs of $L$. erysimi were collected from mustard field and reared on mustard plants in the laboratory. These nymphs were reared on mustard shoots and leaves under ambient environmental conditions at $25 \pm 2{ }^{\circ} \mathrm{C}$. The nymphs of the next generation were used in present study.

Three B.bassiana isolates along with untreated control were evaluated against L. erysimi. There were four replications and thirty nymphs per replica used for the study. Mustard leaves along with shoots were treated with B.bassiana suspension by leaf dip method. Wet filter paper disc $(8.5 \mathrm{~cm}$, Whattman grade $)$ was placed on the bottom of each petri plate before placing treated leaves. A rectangular paraflim was placed under the wet cotton was used to provide water to the leaves as to avoid over-moistening of the filter paper. Thirty aphids were released per replica and the plates were incubated in BOD humidifier at $25 \pm 2{ }^{0} \mathrm{C}$. The petri plates were observed to record the nymphal mortality. The experiment was performed using completely randomized design and the data was analyzed with $\mathrm{LC}_{50}$ in POLO statistical software.

Preparation of sample for Scanning electron microscopy: The SEM studies of fungal isolates of $B$. bassiana were performed on nymph of mustard aphids. Conidial suspension of $B$. bassiana at $10^{9}$ conidia $\mathrm{ml}^{-1}$ was sprayed on nymph stage of $L$. Erysimi after 24, 48, 72 , and 120 hours of incubation respectively. Three to five samples were taken out and processed for SEM studies by using the method of Talaei-hassanloui et al. (2007) with little modifications.

The intact larvae samples were fixed in $2.5 \%$ glutaraldehyde for $24 \mathrm{hrs}$ at $4^{\circ} \mathrm{C}$ followed by rinsing of the sample thrice with Reagent A $(0.1 \mathrm{M}$ cacodylate buffer) for 15 minutes at $4{ }^{\circ} \mathrm{C}$. The secondary fixation of larvae was done with Reagent B (1\% osmium tetra oxide prepared in $0.2 \mathrm{M}$ cacodylate buffer) at $4{ }^{\circ} \mathrm{C}$ for 1 hour. The samples were again washed thrice with Reagent $\mathrm{A}$ at $4{ }^{\circ} \mathrm{C}$ to rinse unfixed osmium tetra oxide. Then the dehydration of the sample was done by passing it through Reagent $C$ involving an ascending ethanol series. The processed nymphs were stubbed and sputter coated in Hitachi gold sputter coater (model

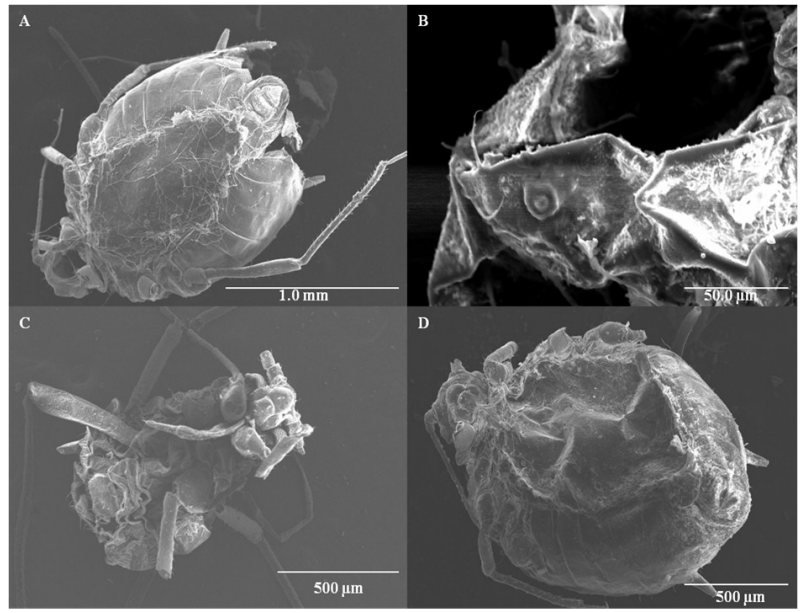

Fig. 1. Scanning EM study of aphid post 24 hours of inoculation A) Fungal hyphae growth on the exoskeleton of aphid in Mycojaal treatment; B)Absence of fungal growth in MTCC 4495; C) No fungal growth observed in fungal isolate F 10; C) Untreated control.

E1010) to view in secondary electron imaging mode in Hitachi S-3400N Scanning Electron Microscope for studying the activity of fungal isolate on infected nymph.

\section{RESULTS AND DISCUSSION}

Scanning electron microscopy of Beauveria bassiana against Lipaphis erysmi KALT: The SEM is one of the most convenient tools to observe the mode of action of entomopathogenic fungi, so that colonization capabilities and extent in rate of infection of the host can be determined (Sun et al., 2016). This tool was used in the present study to investigate the extent and rate of growth of $B$. bassiana on nymphs of $L$. erysimi after first, third, fourth and fifth day of inoculation, so as to compare the biocontrol efficacy of $B$. bassiana MTCC 4495, B. bassiana F10 (Local isolates) and Mycojaal (commercial product of $B$. bassiana) along with untreated control. After $24 \mathrm{hrs}$ of inoculation no fungal growth was observed in $B$. bassiana MTCC 4495 , local fungal isolate F10 and untreated control whereas, commercial formulation isolate Mycojaal showed fungal hyphae growth on the exoskeleton of aphid (Fig. 1). Similar to our results, Li et al. (2016) have observed the adsorption of the conidia and hyphal growth on the surface of the Solenopsis invicta Buren after 2 and 20 hours of inoculation with $1 \times 10^{8}$ conidia $\mathrm{ml}^{-1}$ of $B$. bassiana strain ZGNKY -5. After 72 hours of inoculation, the process of conidiogenesis was observed in Mycojaal. However, penetration of L. ery-

Table 1. The lethal concentration ( $\mathrm{LC}_{-50}$ ) of fungal isolates against L. erysimi.

\begin{tabular}{|c|c|c|c|c|c|c|}
\hline Fungal Isolates & Observations (in hrs) & $\mathrm{LC}_{50}\left(\times 10^{7}\right.$ spores $\left.\mathrm{ml}^{-1}\right)$ & FL & Slope & Chi -square & Hetrogenity \\
\hline \multirow{2}{*}{ MTCC4495 } & 96 & 0.05 & $0.00-9.087$ & $0.157 \pm 0.042$ & 0.789 & 0.78 \\
\hline & 120 & 0.05 & $0.619-5.86$ & $0.349 \pm 0.046$ & 5.80 & 1.93 \\
\hline \multirow{2}{*}{ F10 } & 96 & 0.10 & $0.007-0.152$ & $0.388 \pm 0.054$ & 2.89 & 0.97 \\
\hline & 120 & 0.11 & $0.004-0.56$ & $0.444 \pm 0.057$ & 5.89 & 1.96 \\
\hline \multirow{2}{*}{ Mycojaal } & 96 & 0.04 & $0.00-0.004$ & $0.253 \pm 0.50$ & 0.20 & 0.07 \\
\hline & 120 & 0.06 & $0.00-0.146$ & $0.44 \pm 0.172$ & 0.43 & 0.15 \\
\hline
\end{tabular}




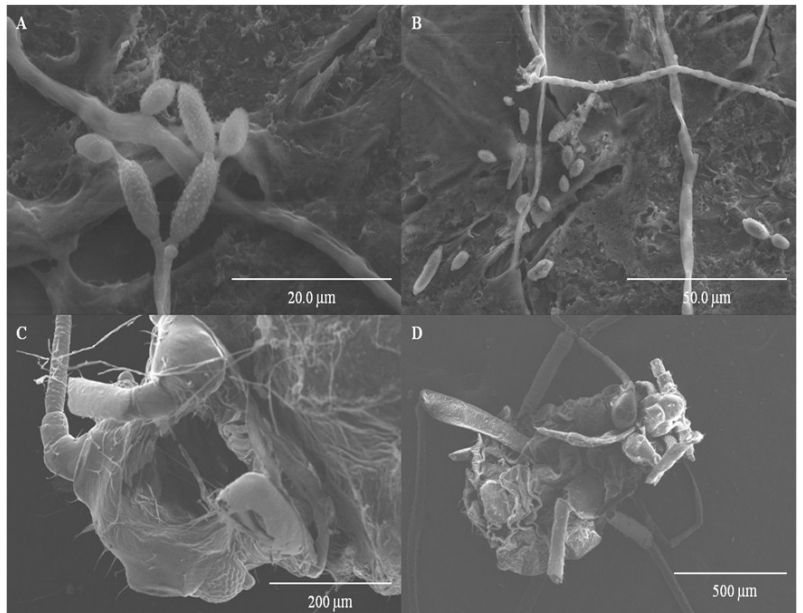

Fig. 2. Scanning EM study of aphid post 72 hours of inoculation A) Presence of conidia (conidiogenesis) on surface of aphid Mycojaal treatment; B) Cuticle penetration by elongated hyphae in MTCC 4495; C) Dislocation of the chitin plates of head showing creation of hollow cavity inside head due to ramification of hyphae and secretion of tissue dissolving enzyme by B. bassiana F10 isolate inside nymph; D) Untreated control.

simi cuticle by elongated hyphae in B. bassiana MTCC 4495 was recorded. A similar result was observed in the form of network of hyphae growing out of the nymph head creating a hollow crescent in the head region for F10 (local isolate of $B$. bassiana) post 72 hours of inoculation and incubation (Fig. 2). The hyphae must have ramified inside the nymph body by generation of secondary hyphae due to germination of spores, formation of appressorium followed by penetration of germ tube in the cuticle. Li et al. (2016) reported formation of the germ tubes of the conidia oriented toward the cuticle after $48 \mathrm{~h}$. The primary vegetative hyphae exhibited formation of elongated chains of two or more conidia as observed under SEM but in untreated control no fungal growth was observed.

After 96 hours of inoculation, mycelium network of Mycojaal isolate covered the whole body of nymph whereas, the presence of conidia, appressorium and germ tube on cuticular surface of $L$. erysimi was observed in B. bassiana MTCC 4495 treated nymphs (Fig. 3). After 120 hours of inoculation and incubation, thick mycelium network covering the outer surface of nymph was also observed in treatment involving inoculation with MTCC 4495 in L. erysimi (Fig.4). Hyphal ramification was recorded throughout the internal surface followed by invasion of outer chitin exoskeleton by the secondary hyphae coming out of the insect internal tissue in fungal isolate F10 after 120 hours. So, little but variation in pathogenesis of Beauveria isolates was recorded at different time interval of fungal infection. A similar SEM study showed variation in efficiency and extent of infection among fungal isolates of B. bassiana than M. anisoplae while little vari-

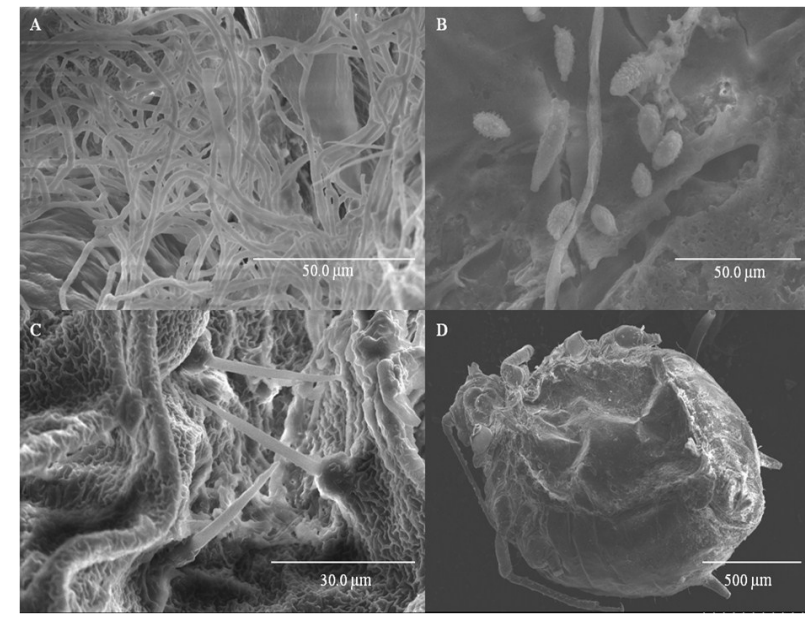

Fig. 3. SEM study of aphid surface post 96 hours of inoculation A) Mycelium network covering the body of nymph in Mycojaal B) Presence of conidia appressorium and germ tube on cuticular surface of L. eysimi in MTCC 4495 C) Growth of B. Bassianaisolate F10 on the surface of nymph;D) ntreated control.

ation was recorded in timing and duration of the phases in the infection process for these two isolates (Moino Jr. et al., 2002). Similar to our observations, Gabarty et al. (2014) reported adhesion and penetration by $B$. bassiana and M. anisopliae on Agrotis ipsilon larvae by SEM. The SE micrographs showed presence of dense hyphal network as well as spores on insect cuticle and also in the body cavity of infected larvae.

Moguel et al. (2013) showed the infection process by Isaria fumosorosea EH-506/3 colonizing whitefly (Trialeurodes vaporariorum) nymphs using light and scanning electron microscopy. The fungal growth development index was used to determine pathogenicity of the test isolates. They recorded colonization of $T$. vaporariorum by the fungus due to formation of cuticular penetration structures and reported that I. fumosorosea produced structures that resembled appressoria in shape causing serious cuticular damage probably via an enzymaticaction and recorded that isolate EH-506/3 was suitable for whitefly biocontrol.

Another SEM report by Talaei-hassanloui et al. (2007) compared the relative efficacy of the uni-, bi- and multi-directional conidial growth i.e. differential polar growth in B. bassiana for imparting pathogenicity. They observed that the unidirectional germination of conidia produced stronger germ tubes and appressorium like structures and thus had highest infection potential as compared to bidirectional and multidirectional conidial growth. Our results also exhibit similar unidirectional germination of the conidia in the local isolate F-10 and the reference culture MTCC 4495.

Lethal concentration $\left(\mathrm{LC}_{50}\right)$ against nymphs of $\boldsymbol{L}$. erysimi: The conidial count required to kill 50 per cent $\left(\mathrm{LC}_{50}\right)$ population of nymph stage of L.erysimi was 


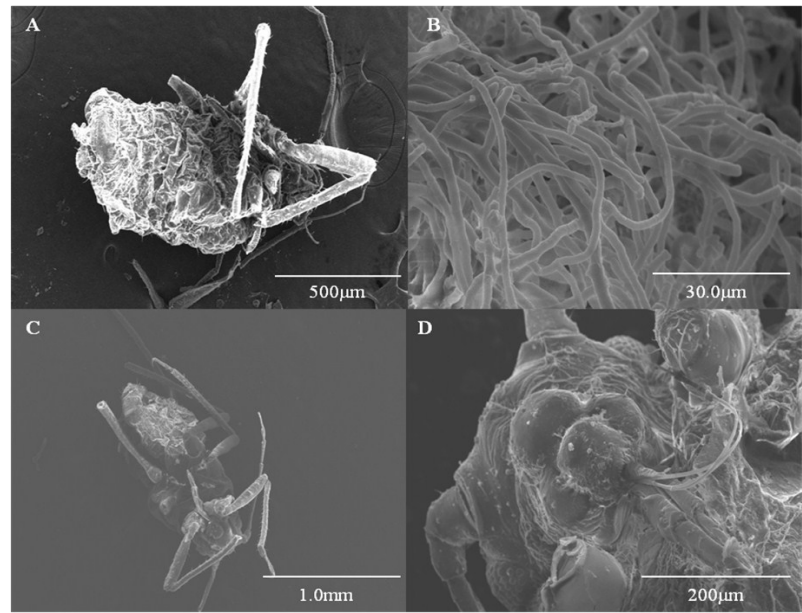

Fig. 4. Scanning EM study of aphid surface post 120 hours of inoculation A) Fungal hyphae growth covering the outer surface in Mycojaal treatment; B) Formation of thick hyphal network on the surface of L. eysimi KALT on inoculation with B. bassiana MTCC 4495; C) Hyphal ramification throughout the internal surface followed by invasion of outer chitin exoskeleton by the secondary hyphae coming out of the insect internal tissue in fungal isolate F10; B) Untreated control.

calculated for all the three B.bassiana isolates. The dosage mortality data obtained was subjected to probit analysis and $\mathrm{LC}_{50}$ values of each fungus was calculated post 96 and 120 hours of inoculation. The $\mathrm{LC}_{50}$ values of fungal isolates of $L$. erysimi ranged from 0.04 to 0.10 after 96 hours of treatment. The highest $\mathrm{LC}_{50}$ value $\left(0.10 \times 10^{7}\right.$ spore $\left.\mathrm{ml}^{-1}\right)$ was recorded in native fungal isolate F10 which showed low effectiveness against $L$. Erysimi whereas, lowest $\mathrm{LC}_{50}$ value $\left(0.04 \times 10^{7}\right.$ spores $\mathrm{ml}^{-1}$ ) was found in commercial isolate of $B$. bassiana (Mycojaal) (Table 1).

After 120 hours of treatment, highest $\mathrm{LC}_{50}$ value $(0.11$ $\mathrm{x} 10^{7}$ spores $\mathrm{ml}^{-1}$ ) was recorded in native fungal isolate $\mathrm{F} 10$ and lowest $\mathrm{LC}_{50}$ value $\left(0.05 \times 10^{7}\right.$ spores $\left.\mathrm{ml}^{-1}\right)$ was recorded in MTCC 4495. Mycojaal isolate and MTCC 4495 were observed to be most effective against $L$. erysimi KALT. Similarly, other scientists also worked on $\mathrm{LC}_{50}$ value of different fungal isolates against different insect pests and reported that the fungal isolates with lowest value of $\mathrm{LC}_{50}$ were more pathogenic. Jiji et al. (2006) evaluated B. bassiana for its potential to control Bactrocera dorsalis and recorded $\mathrm{LC}_{50}$ values of $7.0 \times 10^{8}, 2.0 \times 10^{7}, 5.0 \times 10^{6}$ spores $\mathrm{ml}^{-}$ ${ }^{1}$ on $3^{\text {rd }}, 4^{\text {th }}$ and $5^{\text {th }}$ day of inoculation, respectively. Similarly, Alizadeh et al. (2007) studied the effect of some B. bassiana isolates on Agonoscena pistaciae with $\mathrm{LD}_{50}$ value of $3.91 \times 10^{2}$ and $3.63 \times 10^{4}$ spore per insect.

\section{Conclusion}

The indiscriminate use and over-application of pesticides have serious environmental toxicity concerns which may lead to development of pest resistance, bio- magnification, and harmful impact on non-target organisms. The present study was undertaken to compare and assess the efficacy of three different isolates of entomopathogenic fungus B.bassiana. The results clearly exhibited little but variation in the extent of infection depicted in terms of spore germination in the aphid cuticle post inoculation. The $\mathrm{LC}_{50}$ also varied being lowest (0.04) for commercial formulation $96 \mathrm{hr}$ post inoculation while highest $(0.11)$ for native isolate F10 post 120 hours of inoculation.

\section{ACKNOWLEDGEMENTS}

The authors are thankful to the Head, Department of Entomology and Incharge, Electron Microscopy and Nanoscience Laboratory, Department of Soil Science, Punjab Agricultural University, Ludhiana, Punjab, India for providing the necessary facilities to carry out the present research.

\section{REFERENCES}

Alizadeh, A., Kharrazi Pakdel, A., Talebi-Jahromi, K.H. and Samih, M.A. (2007). Effect of some Beauveria bassiana (Bals). Vull. isolates on common Pistachio Psylla Agonoscena pistaciae Burck. Int. J. Agri. Biol., 9:7679 .

Alves, S.B. (1998). Fungosentomopatogenicos. Alves S B (ed) Controle Microbaino de Insectos. Pp. 289-382. FEALQ, Piracicaba, Brazil.

Cooke, R. C. (1977). Fungi, man and his environment, Longman, London.

Devi, P.S.V., Prasad, Y.G., Chowdary, Y.G.A., Rao, D.M. and Balakrishnan, L.K. (2003). Identification of virulent isolates of the entomopathogenic fungus $\mathrm{Nomu}$ raearileyi (F) Samson for the management of Helicoverpaarmigera and Spodopteralitura. Mycopathol., 156: $365-73$

Gabarty, A., Salem, H.M., Fouda, M.A., Abas, A.A. and Ibrahim, A.A. (2014). Pathogencityinduced by the entomopathogenic fungi Beauveriabassiana and Metarhiziumanisopliae in Agrotisipsilon. $J$ radiation res. and appl sci., 95-100

Gurr, G.M., Wratten, S.D. and Altieri, M. (2004). Ecological Engineering for Pest Management. Advances in Habitat Manipulation for Pest Management. CSIRO Publishing, Melbourne, Australia.

Harrington, R. and Van Emden, H.F. (2007). Aphids as Crop Pests. CABI Publishing, UK.

Jiji, T., Praveena, R., Babu, K., Naseema, A. and Anitha, N. (2006). Three promising fungal strains pathogenic to fruit flies of economic importance from basic to applied knowledge. Proceedings of the 7th International symposium on fruit flies of economic importance. Salvador, Brazil. 175-77

Li, J., Guo, Q., Lin, M., Jiang, L., Ye, J., Chen, D., Li, Z., Dai, J. and Han, S. (2016). Evaluation of a new entomopathogenic strain of Beauveriabassiana and a new field delivery method against Solenopsisinvicta. PLoS ONE 11(6): e0158325. doi:10.1371/journal. pone. 0158325

Luz, C., Tigano, M.S., Silva, I.G., Cordeiro, C.M.T. and Alijanabi, S.M. (1998). Selection of Beauveria bassiana 
and Metarhiziumanisopliae isolates to control Triatomainfestans. Mem.Inst. Oswaldo Cruz., 93:839-846

Moguel, J.C., Mier, T., Montes, M.D.R., Barranco, H.N., Rodriguez, A.Z., Torres, A.P. and Toriello, C. (2013). Fungal growth development index and ultrastructural study of whiteflies infected by three Isariafumosorosea isolates of different pathogenicity. Universidad Nacional Autonoma de Mexico.

Moino, Jr, A., Alves, S.A., Lopes, R.B., Neves, P.M.O.J., Pereira, R.M. and Vieira, S.A. (2002). External development of the entomopathogenic fungi Beauveriabassiana and Metarhiziumanisopliae in the subterranean termite Heterotermes tenuis. Sci. Agric., 59: 267-273

Ormond, E.L., Thomas, A.P.M., Pugh, P.J.A., Pell, J.K. and Roy, H.E. (2010). A fungal pathogen in time and space. The population dynamics of Beauveria bassiana in a conifer forest. FEMS Microbiol. Ecol., 74: 146-54

Quesada-Moraga, E., Landa, B. B., Muñozledesma, J., Jiménez-Díaz, R. M. and Santiago Álvarez, C. (2006). Endo- phytic colonization of opium poppy Papaversomniferum by an entomopathogenic Beauveria bassiana strain. Mycopathol., 161:323-29

Sahoo, S.K. (2012). Incidenc Alizadeh, A., Kharrazi Pakdel, A., Talebi-Jahromi, K.H. and Samih, M.A., ( 2007). Effect of some Beauveria bassiana (Bals). Vull. isolates on common Pistachio Psylla Agonoscena pistaciae Burck. Int. J. Agri. Biol., 9:76-79

Sun, X., Yan, W., Zhang, J., Niu, X., Li, F., Qin, W. and Ma, G. (2016). Frozen section and electron microscopy studies of the infection of the red palm weevil, Rhynchophorusferrugineus (coleoptera:curculionidae) by the entomopathogenic fungus Metarhizium anisopliae. SpringerPlus, 5:1748. DOI 10.1186/s40064-016-3416-6

Talaei-hassanloui, R., Kharazi-pakdel, A., Goettel, M.S., Little, S. and MozaVari, J. (2007). Germination polarity of Beauveria bassiana conidia and its possible correlation with virulence. J. Invert. Pathol., 94:102-107 